\title{
A Simple Procedure for Classical Signal-Processing in Cluster-State Quantum Computation
}

\author{
Kazuto OSHIMA $^{\dagger a)}$,Member
}

SUMMARY We exhibit a simple procedure to find how classical signals should be processed in cluster-state quantum computation. Using stabilizers characterizing a cluster state, we can easily find a precise classical signal-flow that is required in performing cluster-state computation.

key words: cluster state, quantum computation, stabilizer, classical signalflow

\section{Introduction}

Cluster-state quantum computation proposed by Raussendorf and Briegel[1] is a promising scheme for quantum computation. Preparing a cluster-state [2] we can perform quantum computation only by successive quantum measurements and feed forward of measurement outcomes [3]. In cluster state quantum computation it is inevitable to properly choose signs of measurement angles and rectify the resultant quantum state according to preceding random measurement outcomes. Fundamental gates such as the controlled-not gate, an arbitrary single-qubit unitary gate and Hadamard gate have already been studied and given as packages [4]. Combining these packages, an arbitrary computation is carried out. In each package rather complicated eigenvalue equations originating from a cluster-state by successive measurements prescribe how random measurement outcomes affect the sings of measurement angles and an output state. A classical signal-flow in cluster-state computation has also been studied in literature [5]-[9].

Cluster state quantum computation can be simulated by quantum teleportation circuit [10]-[12]. This scheme will give us more flexibility in programming. In this scheme more concise programming and saving the number of qubits will be possible. For example the original package for a controlled-not gate is made of a cluster state with 15 qubits. In contrast with this, in the quantum teleportation scheme this operation can be located on a cluster state as a controlled-phase transformation at the starting point. In the quantum teleportation scheme a correction according to each random measurement outcome is required in the course of computation. In a practical cluster-state quantum computation these corrections should be pushed forward to output qubits. In this process these corrections interact with the Hadamard operator $H$, the rotation operator $Z_{\alpha}=e^{-i \frac{\alpha}{2} Z}$

Manuscript received December 11, 2009.

Manuscript revised January 29, 2010.

†The author is with Gunma National College of Technology, Maebashi-shi, 371-8530 Japan.

a)E-mail: oshima@elc.gunma-ct.ac.jp DOI: 10.1587/transinf.E93.D.1291 and the controlled-phase transformation. These corrections diffuse to other chains through controlled-phase transformations, which causes complexity of a classical-signal flow. Our purpose in this letter is to give a simple procedure to find a precise classical signal-flow in cluster-state quantum computation with the help of the quantum teleportation scheme.

\section{Procedure}

In a quantum teleportation circuit if all measurements results are 0 , we can obtain a correct result without any output corrections or any measurement angle corrections. Therefore if all measurements results are trivial we can perform all controlled-phase transformations before any measurements and we can operate the quantum teleportation circuit only by measuring the corresponding cluster state. As for general measurement outcomes, we can easily see that a measurement result 1 is compensated by an operation $X$ before the meter. Moreover this operation $X$ is compensated by an operation $Z$ on the corresponding qubit in the cluster state. Therefore we can see all effects of the measurement outcomes by the random $Z$ operations on the cluster. We show an example of quantum teleportation circuits and its substitute in Fig. 1. We show that these $Z$ operations can be removed by successive use of stabilizers [13]-[16] characterizing the cluster state.

We consider a cluster state of the following type [2]

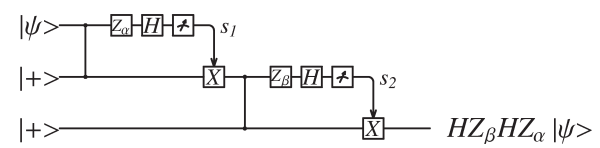

(a)

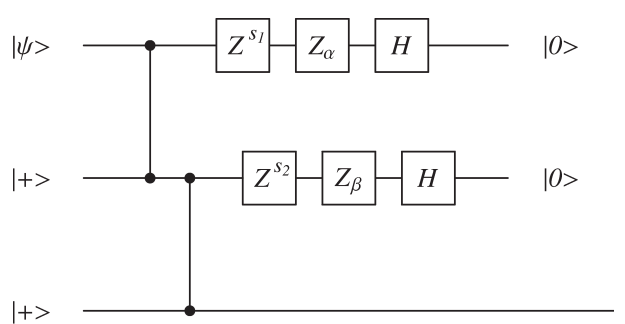

(b)

Fig. 1 (a) Single-qubit two-stage quantum teleportation circuit. The meter means a quantum measurement by the base $\{|0\rangle,|1\rangle\}$. We have two random measurement outcomes $s_{1}, s_{2}$. (b) Corresponding circuit with each random outcome is replaced by the random $Z^{s}$ operator. In compensation all measurement results are assumed to be 0 . 


$$
|\phi\rangle=\Pi_{i}(|0\rangle+|1\rangle \vec{Z})_{i}
$$

where the index $i$ runs over all qubits in the cluster; we assume that this index gives an order for qubits in the cluster and the arrow over the Pauli matrix $Z$ means this operation acts only on adjacent qubits with a number larger than $i$. Here and in the following we neglect an overall factor of a state. This state is characterized by the following stabilizer equations

$$
K_{i}|\phi\rangle=|\phi\rangle,
$$

where $K_{i}=X_{i} \Pi_{j} Z_{j}$ with the index $j$ runs over all adjacent qubits of $i$-th qubit.

We confine ourselves to cluster states composed of plural one-dimensional qubit-chains connected by controlledphase transformations(Fig. 2). It is convenient to divide qubits in a cluster into three parts; input qubits, body qubits and output qubits. Strictly speaking, we do not have a cluster state even at the starting point, because in general input qubits are not in the form $(|0\rangle+|1\rangle \vec{Z})_{i}$. The state $|\Phi\rangle$ we confront is obtained by replacing the state $(|0\rangle+|1\rangle \vec{Z})_{i}$ in $|\phi\rangle$ by an arbitrary state $(a|0\rangle+b|1\rangle \vec{Z})_{i}$ for input qubits in the cluster. This state still satisfies the stabilizer equations $K_{i}|\Phi\rangle=|\Phi\rangle$ for $i$-th qubit not being an input qubit.

As stated before, on account of the randomness of the measurement outcomes, the state $|\Phi\rangle$ changes into the state $\prod_{i} Z_{i}^{s_{i}}|\Phi\rangle$, where the product is for all input qubits and body qubits and $s_{i}$ is a measurement result $0(1)$ of the $i$-th qubit. Let $(i+1)$-th qubit be adjacent to the $i$-th qubit of the same one-dimensional chain in the cluster. Since the operator $K_{i+1}$ has the factor $Z_{i}$, operating $K_{i+1}^{s_{i}}$ on the state $|\Phi\rangle$, we can remove the $Z_{i}^{s_{i}}$ factor on $|\Phi\rangle$. Thus, operating the stabilizers $K_{i}$ on $|\Phi\rangle$ adequately, we can remove all of the $Z_{i}^{s_{i}}$ factors on $|\Phi\rangle$. After these operations we have the following identity

$$
\Pi_{i} Z_{i}^{s_{i}}|\Phi\rangle=\Pi_{j} X_{j}^{f_{j}} Z_{j}^{g_{j}} \Pi_{k} X_{k}^{h_{k}}|\Phi\rangle,
$$

where the index $i$ on the left hand side runs over the input and the body qubits as before and the indices $j$ and $k$ on

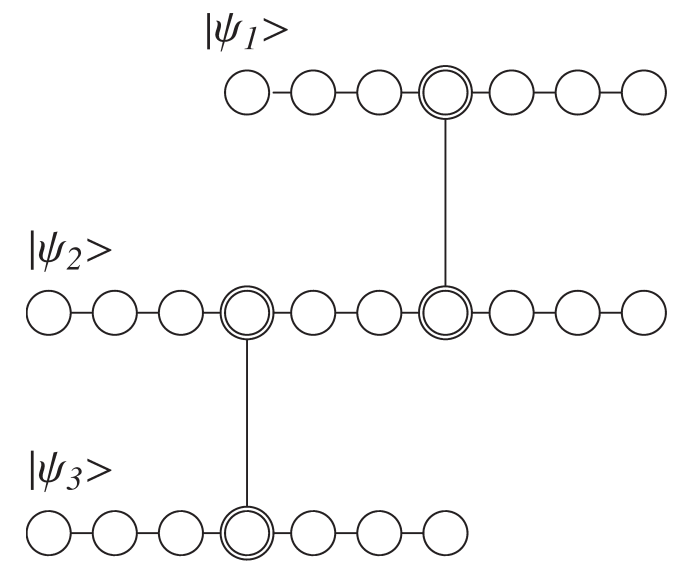

Fig. 2 A typical cluster state composed of plural one-dimensional chains. Vertical lines mean controlled-phase transformations connecting one-dimensional chains. the right hand side run over the output qubits and the body qubits, respectively. The factor $X_{j}^{f_{j}} Z_{j}^{g_{j}}$ is a correction on the $j$-th output qubit depending on the measurement outcomes. Pushing forward the factor $X_{k}^{h_{k}}$ on the $k$-th body qubit to the measurement meter, it changes into a harmless operation $Z_{k}^{h_{k}}$. Since $X^{s} Z_{\alpha}=Z_{(-1)^{s} \alpha} X^{s}$, after the above procedure the measurement angle $\alpha_{k}$ is replaced by $(-1)^{h_{k}} \alpha_{k}$ in the $k$-th qubit. In this way we can easily find the output correction factors $X_{j}^{f_{j}} Z_{j}^{g_{j}}$ and the signs $(-1)^{h_{k}}$ of measurement angles systematically.

\section{Examples}

As an example let us consider an arbitrary single-qubit unitary transformation $U_{z}(\gamma) U_{x}(\beta) U_{z}(\alpha)$. This transformation is carried out by five-qubits cluster state in Fig. 3[4]. The first qubit is an input qubit and the fifth qubit is an output qubit. The measurement angles of the first four qubits are $0, \pm \alpha, \pm \beta, \pm \gamma$, respectively. The \pm factors should be chosen properly depending on the preceding measurement results. We can easily obtain the following identity

$$
\begin{aligned}
& Z_{4}^{s_{4}} Z_{3}^{s_{3}} Z_{2}^{s_{2}} Z_{1}^{s_{1}}|\Phi\rangle \\
= & Z_{4}^{s_{4}} Z_{3}^{s_{3}} Z_{2}^{s_{2}} Z_{1}^{s_{1}} K_{5}^{s_{4}} K_{4}^{s_{3}} K_{3}^{s_{2}} K_{2}^{s_{1}}|\Phi\rangle \\
= & X_{5}^{s_{2}+s_{4}} Z_{5}^{s_{1}+s_{3}} X_{4}^{s_{1}+s_{3}} X_{3}^{s_{2}} X_{2}^{s_{1}}|\Phi\rangle,
\end{aligned}
$$

where we have applied the stabilizer $K_{5}^{s_{4}} K_{4}^{s_{3}} K_{3}^{s_{2}} K_{2}^{s_{1}}$ to remove the factor $Z_{4}^{s_{4}} Z_{3}^{s_{3}} Z_{2}^{s_{2}} Z_{1}^{s_{1}}$. From this identity we find the correction factor of the output qubit $X_{5}^{s_{2}+s_{4}} Z_{5}^{s_{1}+s_{3}}$ and the measurement angles are fixed as $(-1)^{s_{1}} \alpha,(-1)^{s_{2}} \beta,(-1)^{s_{1}+s_{3}} \gamma$.

As for a one-dimensional chain we have seen that it is easy to find a correction factor and to fix the sign factors. We also find that the above procedure can be easily applied to a cluster state containing controlled transformations. Let us consider an H-branch in Fig. 4 that contains

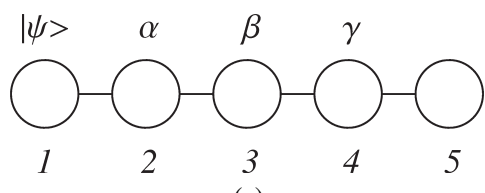

(a)

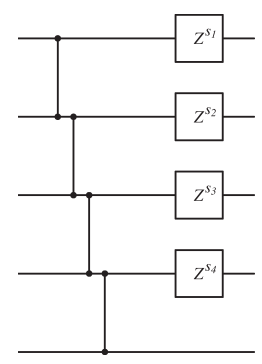

(b)

Fig. 3 (a) One-dimensional cluster state for an arbitrary single-qubit unitary transformation. (b) Corresponding circuit with each random outcome is replaced by the random $Z^{s}$ operator. These $Z^{s}$ operators can be removed by suitable stabilizers. 


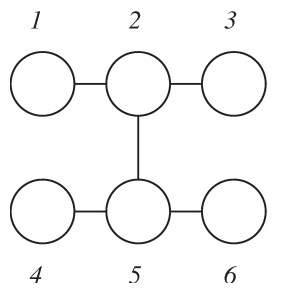

Fig. 4 An H-branch that simulates a controlled transformation.

essential elements for a controlled transformation. We assume that the 1-st qubit and 4-th qubit are input qubits and 3-rd qubit and 6-th qubit are output qubits. Then we have the following state $Z_{5}^{s_{5}} Z_{4}^{s_{4}} Z_{2}^{s_{2}} Z_{1}^{s_{1}}|\Phi\rangle$. To remove the factors $Z_{1}^{s_{1}}, Z_{4}^{s_{4}}$ we first operate the stabilizer $K_{2}^{s_{1}} K_{5}^{s_{4}}$ on $|\Phi\rangle$, where $K_{2}=X_{2} Z_{1} Z_{3} Z_{5}$ and $K_{5}=X_{5} Z_{2} Z_{4} Z_{6}$. After this operation we have $Z_{6}^{s_{4}} Z_{5}^{s_{1}+s_{5}} Z_{3}^{s_{1}} Z_{2}^{s_{2}+s_{4}} X_{5}^{s_{4}} X_{2}^{s_{1}}|\Phi\rangle$. Next to remove the factors $Z_{2}^{s_{2}+s_{4}}, Z_{5}^{s_{1}+s_{5}}$ we operate $K_{3}^{s_{2}+s_{4}} K_{6}^{s_{1}+s_{5}}$ on $|\Phi\rangle$, where $K_{3}=X_{3} Z_{2}$ and $K_{6}=X_{6} Z_{5}$. We finally have the state

$$
Z_{6}^{s_{4}} X_{6}^{s_{1}+s_{5}} Z_{3}^{s_{1}} X_{3}^{s_{2}+s_{4}} X_{5}^{s_{4}} X_{2}^{s_{1}}|\Phi\rangle .
$$

From this expression we see the output correction is $Z_{6}^{s_{4}} X_{6}^{s_{1}+s_{5}} Z_{3}^{s_{1}} X_{3}^{s_{2}+s_{4}}$ and the 2-nd and 5-th qubits' measurement angles are multiplied by the factors $(-1)^{s_{1}}$ and $(-1)^{s_{4}}$. We see that the $Z$-factors of a pair of qubits connected by a vertical line should be removed simultaneously. Our procedure work for cluster states that are composed of H-branches and one-dimensional chains.

\section{Conclusion}

We have given a procedure to process classical signals in the cluster-state quantum computation. We have shown that it is useful to replace random measurement outcomes by random $Z^{s}$ operators. Applying the stabilizers in the proper order to remove the $Z^{s}$ operators, we easily find how classical signals should be processed. Our procedure is very simple since we only have to count the number of the $Z$-factors and to remove them by the stabilizers. Our procedure work well for any cluster-chain that is topologically equivalent to an ordinal quantum circuit.

\section{References}

[1] R. Raussendorf and H.J. Briegel, "A one-way quantum computer," Phys. Rev. Lett., vol.86, no.22, pp.5188-5191, 2001.

[2] H.J. Briegel and R. Raussendorf, "Persistent entanglement in arrays of interacting particles," Phys. Rev. Lett., vol.86, no.5, pp.910-913, 2001.

[3] R. Raussendorf and H.J. Briegel, "Computational model underlying the one-way quantum computer," Quantum Inf. Comput., vol.2, no.6, pp.443-486, 2002.

[4] R. Raussendorf, D.E. Browne, and H.J. Briegel, "Measurementbased quantum computation on cluster states," Phys. Rev. A, vol.68, 022312, 2003.

[5] V. Danos, E. Kashefi, and P. Panangaden, "The measurement calculus," J. ACM, vol.54, no.2(8), pp.1-45, 2007.

[6] V. Danos, E. Kashefi, and P. Panangaden, "Robust and parasimonious ralisations of unitaries in the one-way model," Phys. Rev. A, vol.72, 064301, 2005.

[7] V. Danos and E. Kashefi, "Determinism in the one-way model," Phys. Rev. A, vol.74, 052310, 2006.

[8] N. de Beaudrap, "A complete algorithm to find flows in the oneway measurement model," E-print. http://xxx.lanl.gov/abs/quant-ph/ $0603072,2006$.

[9] N. de Beaudrap, "Finding flows in the one-way measurement model," Phys. Rev. A, vol.77, 022328, 2008.

[10] M.A. Nielsen, "Cluster-state quantum computation." Rep. Math. Phys., vol.57, pp.147-161, 2006.

[11] D. Gottesman and I.L. Chuang, "Demonstrating the validity of universal quantum computation using teleportation and single-qubit operations," Nature, vol.402, pp.390-393, 1999.

[12] E. Knill, R. Laflamme, and G.J. Milburn, Nature, "A scheme for efficient quantum computation with linear optics," vol.409, pp.46$52,2001$.

[13] D. Gottesman, "Stabilizer codes and quantum error correction," Eprint. http://xxx.lanl.gov/abs/quant-ph/9705052/, 1997.

[14] D. Gottesman, "Theory of fault-tolerant quantum computation," Phys. Rev. A, vol.57, pp.127-137, 1998.

[15] A.R. Calderbank, E.M. Rains, P.W. Shor, and N.J.A. Sloane, "Quantum error correction and orthogonal geometry," Phys. Rev. Lett., vol.78, pp.405-408, 1997.

[16] M.A. Nielsen and I.L. Chuang, Quantum Computation and Quantum Information, Cambridge University Press, Cambridge, England, 2000 . 